\title{
Focused Parathyroidectomy without Intraoperative Parathyroid Hormone Testing: Acceptability after Preoperative Localization with SPECT-CT
}

\author{
Titus Cvasciuc ${ }^{1}$, Mark Lansdown ${ }^{2}$, Sheila Fraser ${ }^{3}$
}

\begin{abstract}
Introduction: Intraoperative parathyroid hormone (IOPTH) monitoring is used to confirm the success of parathyroidectomy for primary hyperparathyroidism (PHPT). The aim of this study is to find out if IOPTH is useful during parathyroidectomy for patients with PHPT and positive single photon emission computed tomography (SPECT-CT) imaging.

Materials and methods: A retrospective study of parathyroidectomies for PHPT between 2011 and 2016 in a teaching hospital was performed. Patients' characteristics (demographics and gender), biochemistry, imaging [ultrasound parathyroids (USSs) and SPECT-CT], type of operation, histology, and persistent disease were identified. Patients were divided into four subgroups according to preoperative imaging, use of IOPTH, and failure rate.

Results: Two hundred and fifty-eight patients were enrolled. About $44.6 \%$ of patients had positive and concordant preoperative imaging. Multi-gland disease (MGD) was found in $13.18 \%$ of patients on histology. Patients were divided into four subgroups: group 1 (140 patients): positive imaging and focused parathyroidectomy (FP); group 2 (53 patients): positive imaging and nonfocused approach; group 3 (6 patients): disconcordant imaging [negative SPECT-CT but positive ultrasound (US)] and FP; group 4 (57 patients): negative imaging and no FP. The cure rates were $97.86,94.34,100$, and $87.71 \%$. The overall cure rate was $95 \%$. Within groups 1 and 2 , the failure rate was similar if IOPTH was used. IOPTH was used in all patients in groups 3 and 4.

Conclusion: The selective use of IOPTH is an effective operative strategy. Positive SPECT-CT imaging can guide an FP without the need for IOPTH. IOPTH is useful for SPECT-CT-negative patients who are at risk of multi-gland disease (MGD) or to guide FP if single positive imaging.

Clinical significance: Our results and suggestions can guide the use of IOPTH in clinical practice. The selective use of IOPTH suggested by preoperative imaging can be cost-effective and reduce unnecessary time in theater.

Keywords: Hyperparathyroidism, Intraoperative PTH, Parathyroidectomy.

World Journal of Endocrine Surgery (2019): 10.5005/jp-journals-10002-1245
\end{abstract}

\section{INTRODUCTION}

Primary hyperparathyroidism (PHPT) is the most common cause of hypercalcemia with an incidence varying from 0.4 to 21.6 cases per 100,000 annually. ${ }^{1}$ In $85 \%$ of cases, PHPT is due to a single adenoma. Parathyroid hyperplasia accounts for $5-10 \%$ cases, a double adenoma 3-4\%, and parathyroid carcinoma is extremely rare and causes less than $1 \%$ of all cases of PHPT. $^{2}$

Parathyroidectomy is the only definitive cure and bilateral neck exploration (BNE) was the operative gold standard for many years. ${ }^{3-5}$ However, there has been a shift toward minimally invasive surgery, or a focused parathyroidectomy (FP), as the majority of cases are caused by a single adenoma. Preoperative imaging studies are performed in patients to try to identify a parathyroid adenoma prior to surgery so a focused approach can be used. It is recommended that if a focused approach is planned, at least two imaging studies are performed, with concordant results. Intraoperative parathyroid hormone (IOPTH) has also been previously advocated in this situation.

Negative imaging can occur due to multi-gland disease (MGD), small hypersecreting adenomas, lower levels of serum calcium, and a higher body mass index, which all reduce the sensitivity of imaging. ${ }^{1,6}$ Despite remarkable progress in imaging modalities and surgical management, persistent or recurrent PHPT still occurs in $2.5-5 \%$ of cases ${ }^{7,8}$ with acceptable cure rates between $94 \%$ and $99 \% ., 10$ Ospina et al. in a recent metaanalysis reported that the cure rate was similar between BNE and minimally invasive parathyroidectomy (MIP), with a reported
${ }^{1-3}$ Department of Endocrine Surgery, Leeds Teaching Hospitals, Leeds, Yorkshire, UK

Corresponding Author: Titus Cvasciuc, Department of Endocrine Surgery, Leeds Teaching Hospitals, Leeds, Yorkshire, UK, Phone: +44 7901246047, e-mail: titusicvasciuc@gmail.com

How to cite this article: Cvasciuc T, Lansdown M, Fraser S. Focused Parathyroidectomy without Intraoperative Parathyroid Hormone Testing: Acceptability after Preoperative Localization with SPECT-CT. World J Endoc Surg 2019;11(1):1-5.

Source of support: No external source of support

Conflict of interest: No conflict of interest

98\% cure for BNE and $97 \%$ for MIP, but with lower morbidity for MIP. ${ }^{11}$ The main cause of persistent hypercalcemia was the surgeon's failure to identify abnormal glands and not proceeding to a BNE when there was an inadequate drop in IOPTH levels. ${ }^{12}$

MIP or FP is now an accepted procedure for PHPT. It is preferred by many surgeons and institutions as the initial option for patients with positive preoperative localization tests undergoing the first-time surgery. Contraindications include a family history of parathyroid disease, concurrent thyroid issues (for which, surgical therapy is proposed), and lithium therapy. ${ }^{4,5,13}$

Advocates of IOPTH strongly recommend that it should be used in all patients undergoing FP. ${ }^{14}$ The use of IOPTH improves the outcome of MIP to $99 \%$ success rate if the Miami criteria are respected and if conversion to BNE is performed when IOPTH does

(c) The Author(s). 2019Open Access This article is distributed under the terms of the Creative Commons Attribution 4.0 International License (https://creativecommons. org/licenses/by-nc/4.0/), which permits unrestricted use, distribution, and non-commercial reproduction in any medium, provided you give appropriate credit to the original author(s) and the source, provide a link to the Creative Commons license, and indicate if changes were made. The Creative Commons Public Domain Dedication waiver (http://creativecommons.org/publicdomain/zero/1.0/) applies to the data made available in this article, unless otherwise stated. 
not drop. Ultrasound (US) and parathyroid scintigraphy are the firstline imaging modalities with a sensitivity of $73-90 \%$ and $78-90 \%$, respectively, and a positive predictive value (PPV) of 86 to $93 \%$ and $83-94 \% .{ }^{8}$ Sestamibi scans show up to 97 to $100 \%$ sensitivity and $100 \%$ specificity in localizing enlarged parathyroids. ${ }^{15-17}$

Studies have reported that IOPTH accurately predicts cure in $97-100 \%$ of initial operations and in $78 \%$ of cases of redo surgery. ${ }^{18}$ IOPTH is advised by some authors for all focused redo approaches. ${ }^{7,19}$ The use of IOPTH is not recommended in patients with familial HPT. ${ }^{20}$ Recent studies have described that at 5 minutes, a percentage decline of $35 \%$ of PTH levels best distinguished a single adenoma from MGD (85.3\% vs $24.9 \%){ }^{21}$ These data suggest that when IOPTH does not drop by at least $35 \%$ at 5 minutes postexcision, the surgeon should consider further exploration in the form of a BNE rather than waiting for additional levels. ${ }^{21}$

Opponents of IOPTH monitoring have questioned the benefits of routine IOPTH use and have suggested that the importance diminishes if the parathyroid adenoma is localized by two or more concordant imaging modalities. ${ }^{5,22}$ High-resolution US and technetium-99m sestamibi scintigraphy with SPECT-CT scan have significantly improved the preoperative localization and outcomes of patients undergoing MIP. ${ }^{22}$ However, the true benefit of IOPTH in patients with a preoperatively well-localized single adenoma has been questioned many times in the literature. ${ }^{23,24}$ Recent results from centers that do not use IOPTH demonstrate success rates similar to the institutions that use it routinely. ${ }^{23}$ IOPTH helps confirm a successful parathyroidectomy, but requires more operative time and more resources. ${ }^{5,22,24}$

\section{Materials and Methods}

A retrospective review of all parathyroidectomies performed for PHPT by three surgeons in a UK teaching hospital between January 2011 and December 2016 was conducted. Demographic information, laboratory and imaging study results, surgical procedure, first operation or redo surgery, IOPTH levels, final pathology, and postoperative calcium and PTH levels were collected. Preoperative images were reviewed by operative surgeons, but the study relied on radiology reports for the analysis. Patients with secondary, tertiary hyperparathyroidism as well as those with a history of lithium intake were excluded from this study.

Operative success was defined as normocalcemia at 6 months postoperatively; persistent PHPT was defined as hypercalcemia ( $>2.6 \mathrm{mg} / \mathrm{dL}, 10.4 \mathrm{mg} / \mathrm{dL}$ ) within 6 months of surgery while recurrent hyperparathyroidism was defined as hypercalcemia occurring after 6 months of normocalcemia. For accurate preoperative localization in our practice, we rely on SPECT-CT usually in combination with US examination. IOPTH was usually used when imaging was negative or disconcordant, more often during the second part of our study period and based on surgeons' preference. We used the Miami or modified Miami criteria ( $>50 \%$ drop and/or into the normal range) for IOPTH monitoring. The percentage change in IOPTH was calculated as: $100 \%$ minus (post-excision PTH/pre-excision PTH). PTH specimens were processed using the turbo intact PTH electrochemiluminescence immunoassay and analyzed using a Siemens IMMULITE1000 system (Siemens AG, Erlangen, Germany).

A baseline PTH was checked before incision (pre-incision value), after identifying the enlarged parathyroid but before resection (preexcision), and 10 minutes after resection of the enlarged parathyroid gland(s). According to the Miami criteria, intraoperative success based on IOPTH was defined as $>50 \%$ reduction of the PTH value 10 minutes after removal of the adenoma compared to pre-incision or pre-excision value, whichever was higher. All blood samples were obtained from the same peripheral (brachial) vein. FP (via a lateral or small central approach) was defined as the removal of the enlarged parathyroid gland shown by preoperative imaging without identifying the ipsilateral gland. Frozen section pathology was used to confirm the nature of the tissue removed intraoperatively in selected patients. Positive imaging patients were defined as positive SPECT-CT (and positive USSs if performed). Negative imaging patients were defined as those with negative SPECT-CT imaging. Patients with disconcordant imaging included those with a negative sestamibi component of the SPECT-CT but positive adenoma on CT, or vice versa, or those with a negative SPECT-CT but positive USSs or four dimensional computer tomography (4DCT) neck/thorax. Parathyroid US was used to exclude underlying thyroid diseases or lymph node enlargement and for preoperative US-guided skin marking. Patients had a normal renal function and were fully consented to the procedure. The following SPECT-CT technique was used: GE NM/CT 690 pro with 16-slice diagnostic quality CT; $99 \mathrm{mTc}$-labeled MIBI (adult $900 \mathrm{MBq}$ ) slow intravenous (IV) injection. A high resolution collimator was used with planar images obtained: 10 minutes postinjection $128 \times 128300$ to 600 seconds no zoom, 90 minutes postinjection $128 \times 128300$ to 600 seconds no zoom with SPECT/CT at 90 minutes.

\section{Statistical Analysis}

For statistical calculations, Graph Pad 3.6 State Software, San Diego, California, USA, was used. Nominal variables were described as absolutes and relative frequencies (\%) and the association between them was analyzed by Fisher's exact test. The size effect for statistically significant associations was expressed as an odds ratio (OR) with $95 \%$ confidence interval associated. The level of statistical significance for all two-sided tests was set at $p<0.05$.

\section{Results}

A total of 258 patients ( $81.4 \%$ of females and $18.6 \%$ of males) with biochemically proven PHPT-elevated serum calcium (normal range $2.20-2.60 \mathrm{mmol} / \mathrm{L}$ ) and inappropriately normal or high $\mathrm{PTH}$ levels (normal range 1.5-7.6 pmol/L) - underwent an elective parathyroidectomy for PHPT and were eligible for the study.

The median age in our study group was 56.64 years. The mean preoperative corrected calcium and the PTH level were 2.80 (range 2.32-4.0) $\mathrm{mmol} / \mathrm{L}$ and 25.56 (range 2.7-201.4) pmol/L.

All patients had a SPECT-CT study and 230 patients underwent a parathyroid US preoperatively. About $44.6 \%$ of patients had positive and concordant preoperative SPECT-CT and parathyroid US. About $75.6 \%$ of patients had positive SPECT-CT imaging and, for 47 patients, IOPTH was used additionally. Fifty-seven patients had negative imaging and IOPTH was used for 44 patients in this group.

Thirteen cases $(5 \%)$ had a persistent disease of which six had positive SPECT-CT imaging (five had operation with and one without IOPTH). Seven patients with operative failure were SPECT-CT negative (three had IOPTH and four had no IOPTH measurements). The operative failure rate of SPECT-CT positive vs negative patients was $3.08 \%$ vs $8.89 \%$.

The overall operative success in our study was 95\%. SPECTCT-positive patients had a cure rate of $96.92 \%$, while those with SPECT-CT-negative imaging of $91.11 \%$.

One hundred and forty-six patients underwent FP; 140 had positive, 6 disconcordant imaging (negative SPECT-CT, but positive US), and the majority (108) without IOPTH. One hundred and ten patients had a nonfocused approach and IOPTH was used in 72 patients. 
Single-gland disease was responsible for $84.10 \%$, while parathyroid carcinoma was identified in $2.71 \%$ of our patients.

Patients were divided into four subgroups: group 1 (140 patients): positive imaging and focused approach; group 2 (53 patients): positive imaging and nonfocused approach (uni- or BNE-patients with redo cases, more than one enlarged parathyroid shown on SPECT-CT, suspected cancer cases, intrathyroidal parathyroid glands, simultaneous thyroid operations, and ectopic parathyroid glands not approachable via a focused operation); group 3 (6 patients): disconcordant imaging (negative SPECT-CT but positive US) and FP; and group 4 (57 patients): negative imaging (negative SPECT-CT and US) and non-FP. The groups were compared by IOPTH use and failure rate. Figure 1 shows failure/successful operation within group 1, Figure 2 shows the failure/cure rate within group 2, and Figure 3 shows operative failures/success within group 4.

The cure rate for our groups was $97.86,94.34,100$, and $87.71 \%$.

In group 1, there was no statistical significance regarding persistent disease if we used or not IOPTH ( $p=0.3566)$.

For group 2, IOPTH was used in 28 cases and all 3 patients with persistent disease did not benefit from IOPTH.

There were no failures within group 3 and all six operations were guided by IOPTH.

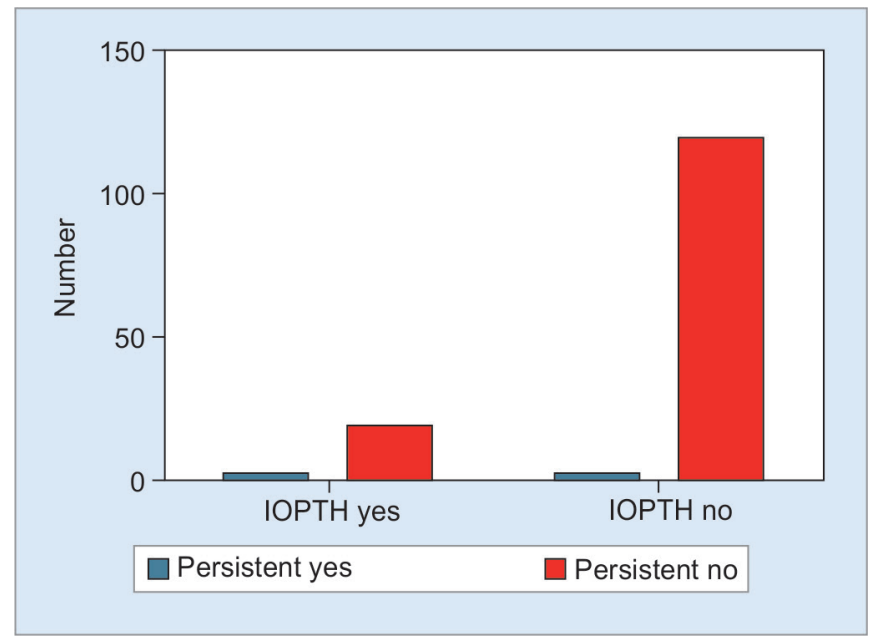

Fig. 1: Failed/successful operation within group 1

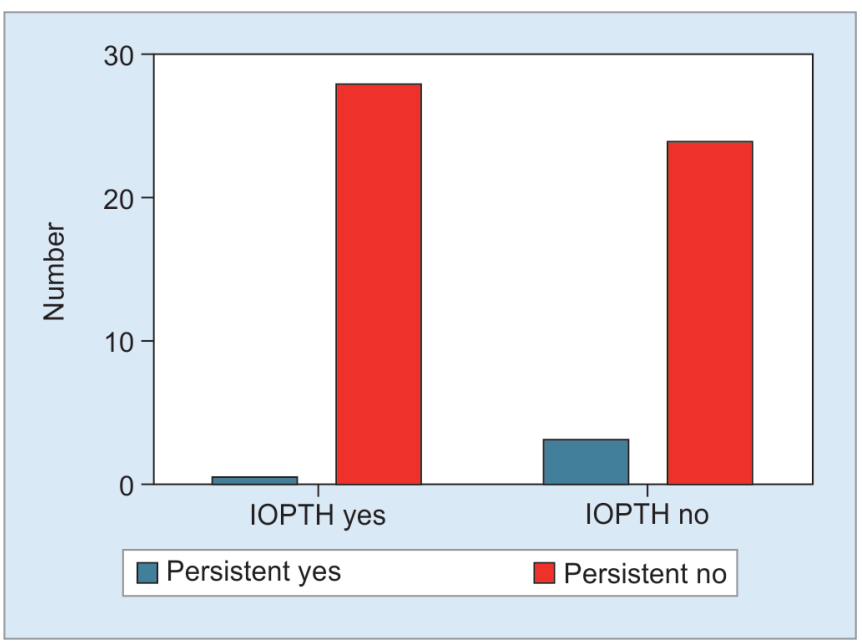

Fig. 2: Failure/cure rate within group 2

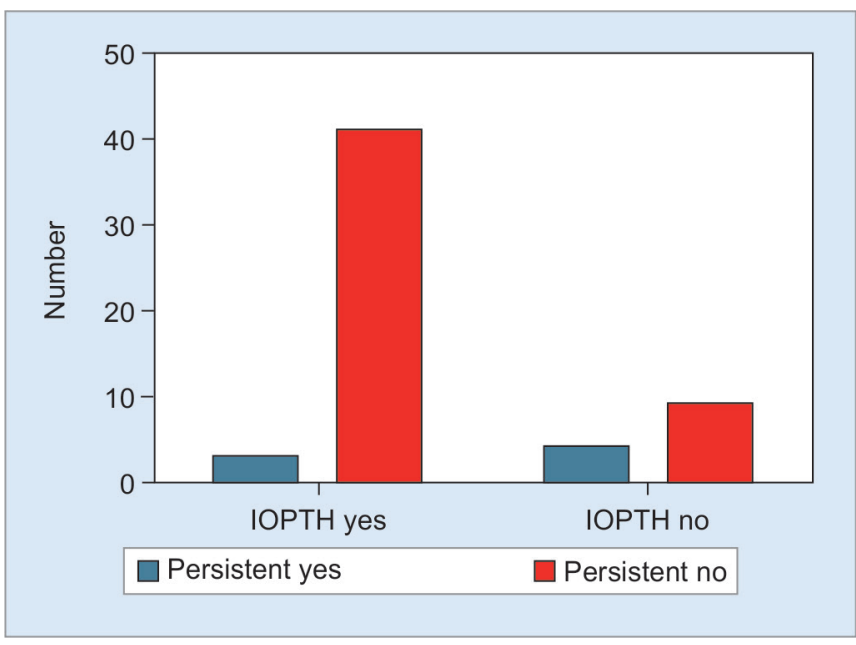

Fig. 3: Operative failures/success within group 4

Group 4 included 57 patients ( 44 with and 13 patients without IOPTH). The failure rate was significantly higher in the subgroup with no IOPTH.

Table 1 shows the patients' characteristics within the groups.

\section{Discussion}

We feel that the routine use of IOPTH for parathyroidectomy is not necessary in appropriately selected patients. ${ }^{6}$ Previous data have shown that even focused surgery with IOPTH can fail and proper patient selection (positive concordant imaging) does not ultimately guarantee success. ${ }^{6}$

The proponents of the routine use of IOPTH quote MGD figures upward of $15 \%,{ }^{6}$ which may not be appreciated preoperatively. MGD is reported in the literature ranging from 4 to $30 \% .^{2}$ In our case series, MGD was encountered in $13.18 \%$ of cases.

It is suggested that the focused approach can be safely and successfully performed without IOPTH for patients with dual positive preoperative imaging studies localizing a single parathyroid adenoma. ${ }^{22}$ The same data showed that patients with one positive imaging study would benefit from IOPTH which demonstrated an increased cure rate from 90 to $96 \%$. $^{22}$

Advocates of IOPTH reported that dual concordant preoperative imaging which detected a single parathyroid adenoma has a PPV of $94 \%$, while additional use of IOPTH led to $100 \%$ cure rates. $^{2}$ Therefore, supporters of IOPTH advise that IOPTH should be used even in patients with concordant preoperative studies. For these authors, IOPTH favors a limited exploration, earlier discharge, reduced complications, and a higher chance of avoiding future reoperations. ${ }^{2}$

In our patient series, when SPECT-CT clearly showed a single enlarged parathyroid, there was no added benefit from adding IOPTH. We found that SPECT-CT in our patient group identified at least one enlarged parathyroid and guided our approach in $75.6 \%$.

There remains controversy regarding the optimal time for taking IOPTH samples. It has been suggested that the optimal time for drawing a pre-incision baseline PTH sample is 10 minutes post-induction of general anesthesia and positioning of the patient. Some authors found a significant difference between pre- and postinduction PTH levels and the Miami criteria were met in $95.45 \%$ of cases with post-induction baseline. ${ }^{16}$ We used a 10 -minute postinduction as a pre-excision sample. Other authors found 20 minutes PTH measurement post-excision to be useful, avoiding unnecessary 
Focused Parathyroidectomy without IOPTH is Acceptable

Table 1: Patients' characteristics within the groups

\begin{tabular}{|c|c|c|c|c|c|c|c|}
\hline Number patients & IOPTH & & Persistent & 95\% confidence interval & $O R$ & pvalue & Cure rate (\%) \\
\hline Group 1 & Yes & 19 & 1 & $0.2848-38.37$ & 3.306 & 0.3566 & 97.86 \\
\hline 140 & No & 121 & 2 & & & & \\
\hline Group 2 & Yes & 28 & 0 & $0.006037-2.498$ & 0.1228 & 0.1115 & 94.34 \\
\hline 55 & No & 27 & 3 & & & & \\
\hline Group 3 & Yes & 6 & 0 & & & & 100 \\
\hline 6 & No & 0 & & & & & \\
\hline Group 4 & Yes & 44 & 3 & $0.03124-0.8677$ & 0.1646 & 0.0407 & 87.71 \\
\hline 57 & No & 13 & 4 & & & & \\
\hline
\end{tabular}

bilateral exploration and the related risk of complications with only a slight increase in the duration of surgery and its costs. ${ }^{13,25,26}$

4D CT can be an alternative to SPECT-CT and has been proven by some authors to predict a good outcome of FP even without using IOPTH but it involves a high dose of irradiation, is expensive, and not available in all centers. ${ }^{19}$

If IOPTH fails to drop by at least $50 \%$, the surgeon should undertake additional neck exploration. ${ }^{23}$ In our study, the postexcision sample was checked at 10 minutes after parathyroid removal and we followed the Miami or modified Miami criteria. If the criteria were not fulfilled at this point, we continued to further explore.

Our results justify the need for IOPTH if there is a high suspicion of MGD. This is consistent with results published elsewhere and is a critical adjunct for the detection of MGD. ${ }^{5}$ Imaging studies recommend where to start the exploration, whereas IOPTH advises when the operation has been successfully accomplished..$^{21,27}$ Similar to other preoperative studies, the best IOPTH results occur in patients with SGD (87-99\% success rate $)^{27}$ and are less accurate in patients with MGD (58\% success rate). ${ }^{1,27}$

In our study, SPECT-CT of the parathyroid glands was a reliable modality to localize parathyroids and was positive in $75.6 \%$ of cases. The majority of SPECT-CT-positive cases underwent focused parathyroidectomies and the cure rate was similar between cases if we used IOPTH or not for single-gland disease with dual concordant imaging. Statistically, there was no better outcome if we used IOPTH for this subgroup of patients.

For group 2, IOPTH should be used judiciously provided that the intraoperative findings correlate with preoperative imaging findings. Even if we have positive concordant preoperative imaging, we preferred a nonfocused approach for patients with large/giant adenomas, more than one suggested enlarged parathyroids, previous neck surgery, suspicion of ectopic gland (intrathyroidal) parathyroid carcinoma, or additional thyroid pathology.

Group 3 identifies only a small number of patients and we advise that the operative strategy should be always guided by IOPTH.

There was a statistical difference when using IOPTH for group 4. Our recommendation is that all patients with negative imaging should have used IOPTH to improve the outcome of a parathyroidectomy. In Flowchart 1, we highlight the algorithm of managing PHPT at our institution.

The percentage of positive and concordant studies varies in different studies, ranging from 55 to $64 \% .{ }^{27}$ In total, $44.5 \%$ of our patients had both positive and concordant images, as seen on SPECT-CT with both the sestamibi and the CT component. IOPTH can successfully guide the operation in patients with nonconcordant studies in about $74 \%$ of patients. ${ }^{27}$

An US of the parathyroids was usually performed in our institution to rule out any concurrent thyroid pathology or indeterminate cases on SPECT-CT, rather than to initially localize a parathyroid adenoma. Coexistence of thyroid nodules is found in 20 to $60 \%$ of patients with primary $\mathrm{HPT}^{28}$ so an US of the parathyroids is carried out before we plan parathyroid surgery to detect any indeterminate/suspicious thyroid nodules or intrathyroidal parathyroids. ${ }^{29}$

The European Society of Endocrine Surgeons stated that IOPTH is useful for patients undergoing parathyroidectomy on the basis of a single preoperative localization study if preoperative localization with MIBI and US is not concordant. However, if preoperative dual localization with MIBI and US is concordant for single-gland disease, the use of

Flowchart 1: Highlighting the algorithm of managing PHPT at our institution

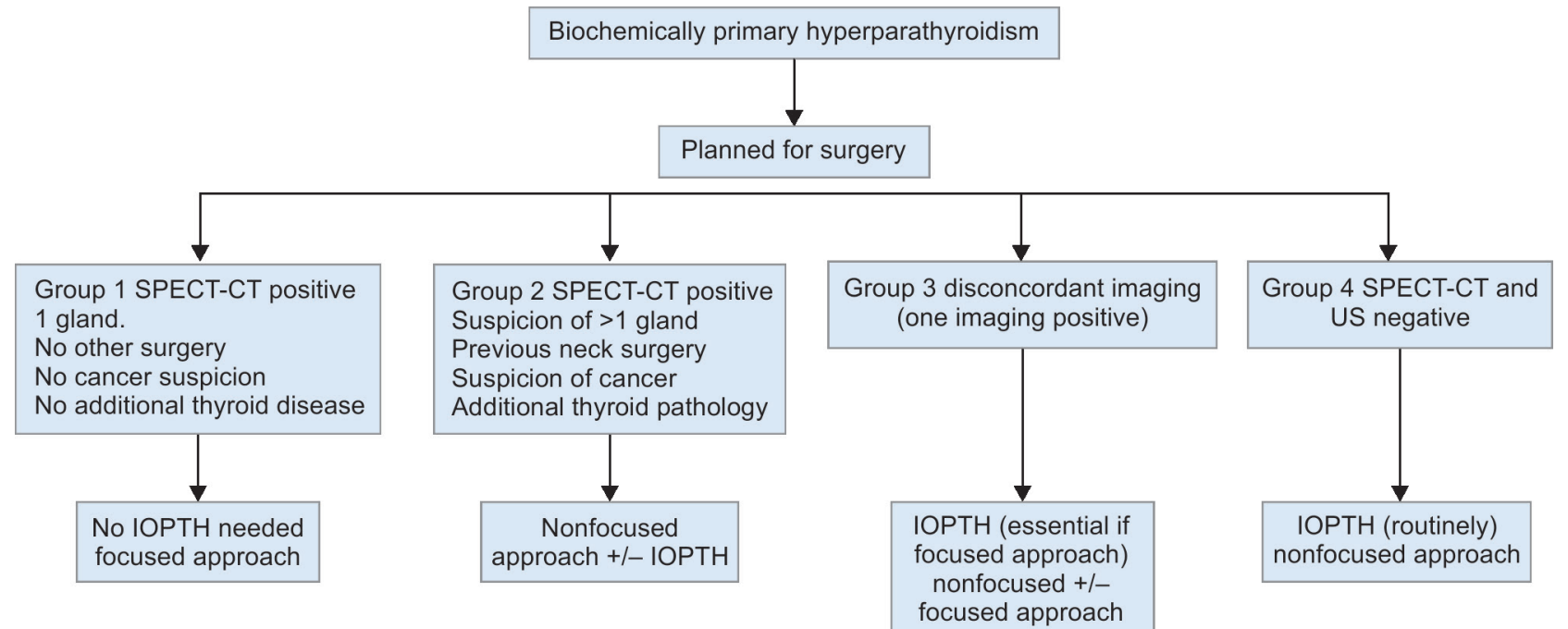


IOPTH is of minimal value. ${ }^{13}$ In our department, a single preoperative localization study with SPECT-CT parathyroids can guide our operative approach. We find it very useful to discuss this imaging preoperatively with radiologists in a multidisciplinary meeting if there are any doubts or indeterminate imaging. We use 4D CT on a selective basis.

Opponents of routine IOPTH use suggest that there is little benefit in the presence of highly sensitive dual preoperative localization studies. In addition, IOPTH monitoring prolongs the procedure by at least 30 minutes and has additional costs. ${ }^{14,30}$ The use of IOPTH increases the overall cost by $4 \%$ for a focused approach. ${ }^{27}$ Alternatively, IOPTH is an objective and quantifiable outcome and can be used to compare outcomes between institutions. ${ }^{10}$

\section{ConcLusion}

We feel that the selective use of IOPTH is an effective strategy. Patients with positive dual preoperative imaging will benefit from a focused approach without IOPTH monitoring. IOPTH is useful for patients with negative imaging, who are at a higher risk of MGD or to guide a focused approach if imaging is disconcordant.

\section{Clinical Significance}

Our results can guide the use of IOPTH in clinical practice. Selective use of IOPTH suggested by preoperative imaging is appropriate, cost-effective, and reduces operative time.

\section{References}

1. Wachtel H, Cerullo I, et al. What can we learn from intraoperative parathyroid hormone levels that do not drop appropriately? Ann Surg Oncol 2015;22:1781-1788. DOI: 10.1245/s10434-014-4201-9.

2. Bobanga ID, McHenry CR. Is intraoperative parathyroid hormone monitoring necessary for primary hyperparathyroidism with concordant preoperative imaging? Am J Surg 2017;213(3):484-488. DOI: 10.1016/j.amjsurg.2016.11.035.

3. Dobrinja C, Santandrea G, et al. Effectiveness of intraoperative parathyroid monitoring (ioPTH) in predicting a multiglandular or malignant parathyroid disease. Int J Surg 2017;41Suppl 1:S26-S33. DOI: 10.1016/j.ijsu.2017.02.063.

4. Nelson CM, Victor NS. Rapid intraoperative parathyroid hormone assay in the surgical management of hyperparathyroidism, Perm J 2007 Winter;11(1):3-6.

5. Khan AA, Khatun Y, et al. Role of intraoperative PTH monitoring and surgical approach in primary hyperparathyroidism. Ann Med Surg 2015;4(3):301-305. DOI: 10.1016/j.amsu.2015.08.007.

6. Thielmann A, Kerr P. Validation of selective use of intraoperative PTH monitoring in parathyroidectomy. J Otolaryngol Head Neck Surg 2017;46(1):10. DOI: 10.1186/s40463-017-0188-0.

7. Guerin C, Paladino NC, et al. Persistent and recurrent hyperparathyroidism. Updates Surg 2017;69(2):161-169. DOI: 10.1007/ s13304-017-0447-7.

8. Yeh MW, Wiseman JE, et al. Population-level predictors of persistent hyperparathyroidism. Surgery 2011;150(6):1113-1119. DOI: 10.1016/j. surg.2011.09.025.

9. Hessman O, Stalberg P, et al. High success rate of parathyroid reoperation may be achieved with improved localization diagnosis. World J Surg 2008;32(5):774-781. DOI: 10.1007/s00268-008-9537-5.

10. Lew Jl, Solorzano CC. Surgical management of primary hyperparathyroidism: state of the art. Surg Clin North Am 2009;89:1205-1225. DOI: 10.1016/j.suc.2009.06.014.

11. Singh Ospina NM, Rodriguez-Gutierrez R, et al. Outcomes of parathyroidectomy in patients with primary hyperparathyroidism: a systematic review and meta-analysis. World J Surg 2016;40(10): 2359-2377. DOI: 10.1007/s00268-016-3514-1.

12. Lee $\mathrm{S}$, Ryu $\mathrm{H}$, et al. Operative failure in minimally invasive parathyroidectomy utilizing an intraoperative parathyroid hormone assay. Ann Surg Oncol 2014;21(6):1878-1883. DOI: 10.1245/s10434-013-3479-3.

13. Bergenfelz AOJ, Hellman P, et al. Positional statement of the European Society of Endocrine Surgeons (ESES) on modern techniques in pHPT surgery. Langenbecks Arch Surg 2009;394:761-764. DOI: 10.1007/ s00423-009-0533-5.

14. Chen $\mathrm{H}$, Pruhs $\mathrm{Z}$, et al. Intraoperative parathyroid hormone testing improves cure rates in patients undergoing minimally invasive parathyroidectomy. Surgery 2005;138(4):583-587. DOI: 10.1016/j. surg.2005.06.046.

15. Caixàs A, Bernà L, et al. Efficacy of preoperative diagnostic imaging localization of technetium $99 \mathrm{~m}$-sestamibi scintigraphy in hyperparathyroidism. Surgery 1997;121(5):535-541.

16. Malhotra A, Silver C, et al. Preoperative parathyroid localization with sestamibi. Am J Surg 1996;172:637-640.

17. O'Doherty MJ, Kettle AG, et al. Parathyroid imaging with technetium99m-sestamibi: preoperative localization and tissue uptake studies. J Nucl Med 1992;33(3):313-318.

18. Richards ML, Thompson GB, et al. Reoperative parathyroidectomy in 228 patients during the era of minimal-access surgery and intraoperative parathyroid hormone monitoring. Am J Surg 2008;196(6):937-942. DOI: 10.1016/j.amjsurg.2018.12.057.

19. Heineman TE, Kutler DI, et al. Is intraoperative parathyroid hormone monitoring warranted in cases of $4 \mathrm{D}-\mathrm{CT} / \mathrm{ult}$ trasound localized single adenomas? Otolaryngol Head Neck Surg 2015;153(2):183-188. DOI: 10.1177/0194599815590597.

20. Guerrero $\mathrm{MA}$, Clark $\mathrm{OH}$. A comprehensive review of intraoperative parathyroid hormone monitoring. World J Endocr Surg 2010;2(1): 21-27.

21. Alhefdhi $A$, Ahmad $K$, et al. Five minute intraoperative parathyroid hormone levels can identify multigland disease. Ann Surg Oncol 2017;24(3):733-738. DOI: 10.1245/s10434-016-5617-1.

22. Zawawi F, Mlynarek AM, et al. Intraoperative parathyroid hormone level in parathyroidectomy: which patients benefit from it? J Otolaryngol Head Neck Surg 2013;42(1):56. DOI: 10.1186/19160216-42-56.

23. Najafian A, Kahan S, et al. Intraoperative PTH may not be necessary in the management of primary hyperparathyroidism even with only one positive or only indeterminate preoperative localization studies. World J Surg 2017;41(6):1500-1505. DOI: 10.1007/s00268-017-3871-4.

24. Shawky MS. Intraoperative parathyroid hormone monitoring in primary hyperparathyroidism; resolving controversies and debates. J Parathyroid Dis 2017;5(1);3-10.

25. Garbutt L, Sigvaldason H, et al. What is the most appropriate intraoperative baseline parathormone? A prospective cohort study. Int J Surg 2016;25:49-53. DOI: 10.1016/j.ijsu.2015.11.044.

26. Calò PG, Pisano G, et al. Intraoperative parathyroid hormone assay during focused parathyroidectomy: the importance of 20 minutes measurement. BMC Surg 2013 Sep 18;13:36. DOI: 10.1186/1471-248213-36.

27. Perrier ND, Ituarte PHG, et al. Parathyroid surgery: separating promise from reality. J Clin Endocrinol Metab 2002;87(3):1024-1029. DOI: 10.1210/jcem.87.3.8310.

28. Strichartz SD, Giuliano AE. The operative management of coexisting thyroid and parathyroid disease. Arch Surg 1990;125:1327-1331.

29. Kim HG, Kim WY, et al. Minimally invasive parathyroidectomy with or without intraoperative parathyroid hormone for primary hyperparathyroidism. Ann Surg Treat Res 2015;89(3):111-116. DOI: 10.4174/astr.2015.89.3.111.

30. Wong W, Foo FJ, et al. Simplified minimally invasive parathyroidectomy: a series of 100 cases and review of the literature. Ann R Coll Surg Engl 2011;93:290-293. DOI: 10.1308/003588411X571836. 